\title{
PRIME KNOTS OBTAINED BY BAND SUMS
}

\author{
Mario Eudave-MuÑoz
}

\begin{abstract}
We consider the primeness of knots obtained by band sums, and prove that under certain circumstances nontrivial band sums of knots yield prime knots.
\end{abstract}

For $k_{0}$ and $k_{1}$ knots in $S^{3}$, let the band sum of $k_{0}$ and $k_{1}$ be defined as follows: imbed $k_{0}$ and $k_{1}$ into disjoint 3-balls in $S^{3}$; let $b: I \times I \rightarrow S^{3}$ be an imbedding such that $b^{-1}\left\{k_{i}\right\}=I \times\{i\}, i=0,1$; then join $k_{0}-b(I \times\{0\})$ to $k_{1}-b(I \times\{1\})$ by the arcs $b(\partial I \times I)$. The resulting knot is the band sum of $k_{0}$ and $k_{1}$ along $b$ and is denoted $k_{0} \#_{b} k_{1}$. A sphere in $S^{3}$ disjoint from $k_{0}$ and $k_{1}$ which separates them is called a splitting sphere. A band sum is trivial if it is equivalent to a connected sum, or what is the same, there is a splitting sphere which meets the band exactly in one arc parallel to $b(I \times\{1 / 2\})$.

A knot $k$ is composite if there is a sphere $P$ intersecting transversally $k$ in two points, such that each one of the 3-balls bounded by $P$ intersects $k$ in a knotted spanning arc. Such a sphere is called a decomposing sphere for $k$. A knot is prime if it is neither composite, nor trivial.

Let $K=k_{0} \#_{b} k_{1}$. If the band is trivial then $k$ will be composite unless $k_{0}$ or $k_{1}$ is a trivial knot. Suppose the band is not a trivial one. If $k_{0}$ is a composite knot and one of its summands is not perturbed by the band, i.e., there is a decomposing sphere for $k_{0}$ which is disjoint from the band, then this summand will remain in $K$ and so $K$ will be a composite knot. On the other hand if $K$ is composite and there is a decomposing sphere for $K$ disjoint from the band, then this sphere will be also a decomposing sphere for $k_{0}$ or $k_{1}$. It is possible for $K$ to be composite even if $k_{0}$ and $k_{1}$ are not; for example the square knot is a band sum of trivial knots. Under certain circumstances $K$ may be prime, as is shown in the following theorem.

THEOREM 1. Let $K=k_{0} \#_{b} k_{1}$ be a nontrivial band sum of prime knots $k_{0}$ and $k_{1}$. Suppose the band is disjoint from one incompressible Seifert surface for $k_{0} \cup k_{1}$. Then $K$ is prime. 
REMARKS. (1) A slightly stronger statement is actually proven: If $k_{0}$ and $k_{1}$ are not necessarily prime knots, then either $K$ is prime, or there is a decomposing sphere for $K$ disjoint from the band.

(2) If $\operatorname{genus}(K)=\operatorname{genus}\left(k_{0}\right)+\operatorname{genus}\left(k_{1}\right)$, then there is a minimal genus Seifert surface for $k_{0} \cup k_{1}$ disjoint from the band [Ga], [ $\mathbf{S c}_{3}$, 8.4].

Let $K=k_{0} \#_{b} k_{1}$ be a nontrivial band sum. We would like to determine exactly when $K$ is a composite knot. The solution of the following conjecture would help.

CONJECTURE. If $K$ is composite then there is a decomposing sphere which intersects the band exactly in one arc parallel to $b(\{1 / 2\} \times I)$, or there is a decomposing sphere disjoint from the band.

There are examples which support this conjecture; for instance the square knot is a band sum of trivial knots, and there is a decomposing sphere crossing the band once. S. A. Bleiler and M. Eudave-Muñoz [BE] have proved the following result: if $K=k_{0} \#_{b} k_{1}$ is composite and $k_{0}$ and $k_{1}$ are trivial knots, then one of the summands of $K$ is a 2-bridge knot. This result would follow directly from the conjecture.

Proof of Theorem 1. Suppose $S$ is an incompressible Seifert surface for $k_{0} \cup k_{1}$ disjoint from the band. Take a splitting sphere $Q$ which among all such spheres, has a minimal number of arcs of intersection with the band. Because $S$ is incompressible, it can be made disjoint from $Q$, so $S=S_{0} \cup S_{1}$, where $S_{i}$ is an incompressible Seifert surface for $k_{i}, i=0,1$. Then $T=S \cup b(I \times I)$ is a Seifert surface for $K$.

It follows from the incompressibility of $S_{0}$ and $S_{1}$ and the choice of $Q$ that $Q \cap T=Q \cap b(I \times I)=$ odd number of arcs. Let $m$ be this number of arcs. $T$ is incompressible. We will assume this here, for the proof is similar to that below, but easier-it requires only applying cases (1) and (2) below to the intersections of $Q$ with a possible compression disk for $T$.

Suppose that $K$ is composite, and $P$ is a decomposing sphere. By the incompressibility of $T$ we can suppose that $T \cap P=\gamma=$ one arc; and we can also suppose that $P \cap b(I \times I)=\left\{b\left(x_{i} \times I\right)\right\}$, for some finite collection of points $\left\{x_{i}\right\}$. Take a decomposing sphere which has a minimal number $n$ of arcs of intersection with the band, call it $P$. If $n=0$ then either $k_{0}$ or $k_{1}$ are not prime, or the band is trivial, so suppose $n \neq 0$. Consider a regular neighborhood $\eta(T)$ of $T$. Call $T_{+}$and $T_{-}$the top and bottom parts of $\partial \eta(T)$ respectively. 
Let $M=S^{3}-\operatorname{int}(\eta(T))$. Then $P^{\prime}=P \cap M$ and $Q^{\prime}=Q \cap M$ are planar surfaces in $M . P^{\prime}$ and $Q^{\prime}$ are incompressible in $M$, by the minimality of $m$ and $n$. We can suppose that there are no inessential circles of intersection between $P^{\prime}$ and $Q^{\prime} . \partial Q^{\prime}$ is a collection of $m$ curves parallel to $b(I \times\{1 / 2\})$, which go once over $T_{+}$and once over $T_{-}$; label these curves as $a_{1}, \ldots, a_{m} . \partial P^{\prime}$ consists of two arcs, one arc lying in $T_{+}$and the other in $T_{-}$; call them $\gamma_{+}$and $\gamma_{-}, \gamma_{+} \cup \gamma_{-}$intersects the neighborhood of the band in $n$ pair of arcs; call them $b_{1}, b_{2}, \ldots, b_{n}$. The circle $a_{i}$ meets each of the two arcs $b_{j} \subset \partial P^{\prime}$ in a single point; label these crossings with $i$ in $P^{\prime}$ and with $j$ in $Q^{\prime}$. Consider graphs in $P^{\prime}$ and $Q^{\prime}$ as in [S $\left.\mathbf{s}_{1}, \S 2\right]$.

$P^{\prime}$ is a disk, so the corresponding graph looks like a collection of arcs with both ends in $\partial P^{\prime}$. Take an innermost arc $\alpha$ in $P^{\prime}$; this $\alpha$ determines a disc $D$ in $P^{\prime}$ with interior disjoint from $Q^{\prime}$. There are the following cases.

(1) $\alpha$ has its ends in $\gamma_{+}\left(\gamma_{-}\right)$, and are labelled $i, i+1$. It is not difficult to see that with the disc $D$ and the annulus cobounded by $a_{i}$ and $a_{i+1}$ in $\partial \eta(T)$, a compression disk for $Q^{\prime}$ is constructed.

(2) $\alpha$ has both ends in $\gamma_{+}$(or both in $\gamma_{-}$), and are labelled 1,1 (or $m, m)$.

$\partial D=\alpha \cup \beta$, where $\beta$ is an arc contained in $T$ which lies in $b(I \times I) \cup$ $S_{0}$ (or $S_{1}$ ). The corresponding $\operatorname{arc} \alpha$ in $Q^{\prime}$ is a loop with ends in $a_{1}$; take the component $F$ of $Q^{\prime}$ determined by $\alpha$ such that $\partial F=\alpha \cup \delta, \delta \subset T_{+}$. $R=D \cup F$ is a planar surface; one component of $\partial R$ is $\beta \cup \delta$. By doing an isotopy we can suppose $\beta \cup \delta \subset S_{0_{+}}$; consider $E=R \cup$ \{disks determined by the $a_{i}$ 's which are contained in $\left.F\right\} . E$ is a disk in $S^{3}-\eta\left(S_{0}\right)$ with boundary in $S_{0_{+}}$. Because $S_{0}$ is incompressible, $\partial E$ must bound a disk in $S_{0}$, so $\beta \cup \delta$ bounds a disk. But this implies it is possible to isotope $P$ to reduce the number of arcs of intersection with the band, which is a contradiction.

(3) $\alpha$ has one end in $\gamma_{+}$and the other in $\gamma_{-}$, both labelled 1,1 $(m, m)$.

Because cases (1) and (2) are not possible, all the arcs in $P^{\prime}$ are parallel to $\alpha$ and each one of these arcs has its ends equally labelled. Then in $Q^{\prime}$ all the arcs are loops with ends equally labelled. Take one innermost, and let this be $\beta$; using the disk determined by $\beta$ we can do surgery on $P^{\prime}$ to obtain another decomposing sphere with fewer intersection arcs with the band [E, 2.6], which is a contradiction. 
These are all the possible cases because $\partial P^{\prime}$ intersects $Q$ only over the band, and because $T-b$ is disconnected. So the proposition is proved.

We would try to prove the conjecture in the same way as Theorem 1. By [Ga] there is a minimal genus Seifert surface for $K$ which contains the band. We have the following cases: (a) The Seifert surface minus the band is incompressible, this is the case of Theorem 1. (b) The Seifert surface minus the band is compressible; then a splitting sphere may intersect the Seifert surfaces in essential circles, and the arc of intersection between the Seifert surface and a decomposing sphere may intersect the splitting sphere outside of the band, creating another kind of innermost loop. Unfortunately such an innermost loop is not always removable.

Let $K=k_{0} \#_{b} k_{1}$ be a band sum. Suppose there are Seifert surfaces $S_{0}$ and $S_{1}$ for $k_{0}$ and $k_{1}$ contained in disjoint balls, which are disjoint from the band. Let $S=S_{0} \cup S_{1} \cup b(I \times I)$, and $M=S^{3}-\eta(S)$.

THEOREM 2. If there is a compressing disk for $\partial \eta(S)$ in $M$ whose boundary intersects $K$ in at most $2 n$ points, then either it can be isotoped off the band or there is a compressing disk disjoint from the band whose boundary intersects $K$ in at most $n+1$ points.

Proof. Suppose $P$ is a compressing disk for $\partial \eta(S)$ whose boundary intersects $K$ in $2 n$ points, and intersects the band in $m$ arcs; isotope $P$ so that $2 n+m$ is minimum. Let $Q$ be a splitting sphere for $k_{0} \cup k_{1}$ intersecting the band in a minimal number of arcs. Let $Q^{\prime}=Q \cap M$; consider in $Q^{\prime}$ the same structure as its analogue has in the proof of Theorem 1. Consider the intersections between $P$ and $Q^{\prime}$. If an innermost arc in $P$ has its ends differently labelled, then we have the same situation as case (1) above, so suppose all the innermost arcs have its ends equally labelled. If all the arcs are parallel in $P$, each label of $Q^{\prime}$ appears at both ends of one of these arcs. If there are more than two innermost arcs, take an outermost fork in $P$ (as defined in [S. $\left.\mathbf{S c}_{2}\right]$ ); this also implies each label of $Q^{\prime}$ appears at both ends of one arc, so in $Q^{\prime}$ each vertex is the base of a loop. Take an innermost loop in $Q^{\prime}$; let this be $\beta$. The boundary of the disk determined by $\beta$ in $Q^{\prime}$ intersects $K$ at most in two points. Doing surgery in $P$ with this disk we get two disks $P_{1}$ and $P_{2}$. If one of them is not a compressing disk, then it is possible to isotope $P$ reducing $2 n+m$. Since this is not possible, both of them are compressing disks, one of them intersects $K$ in $\leq n+1$ points, and both can be isotoped to intersect the band in less 
than $m$ arcs. Continuing in this way we can find a compressing disk disjoint from the band, and intersecting $K$ in $\leq n+1$ points. This also implies that $\partial \eta(S)$ is incompressible in $M$ or there is a compressing disk disjoint from the band.

COROLlaRY. If $\partial \eta\left(S_{0}\right)$ and $\partial \eta\left(S_{1}\right)$ are incompressible, then either $\partial \eta(S)$ is incompressible or the band sum is a connected sum.

I would like to thank Marty Scharlemann for his suggestions.

\section{REFERENCES}

[BE] S. A. Bleiler and M. Eudave-Muñoz, Composite ribbon number one knots have two bridge summands, preprint.

[E] M. Eudave-Muñoz, Primeness and sums of tangles, Trans. Amer. Math. Soc., 306 (1988), 773-790.

[Ga] D. Gabai, Genus is superadditive under band connected sum, Topology, 26 (1987), 209-210.

[Sc $\mathrm{Sc}_{1}$ M. Scharlemann, Smooth spheres in $R^{4}$ with four critical points are standard, Inv. Math., 79 (1985), 121-141.

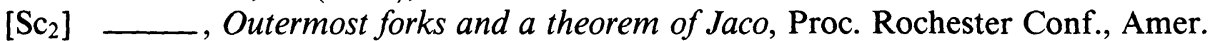
Math. Soc. Contemporary. Math. Series, 44 (1985), 189-193.

[ $\left.\mathrm{Sc}_{3}\right]$ - Sutured manifolds and generalized Thurston norms, to appear in $\mathrm{J}$. Differential Geom.

Received January 20, 1988 and in revised form March 19, 1988. Research supported in part by a grant from the National Science Foundation.

University of California, Santa Barbara

SANTA BARBARA, CA 93106 
